\title{
Effects of practice and distribution of auditory signals on absolute identification
}

\author{
DANIEL L. WEBER, DAVID M. GREEN, and R. DUNCAN LUCE \\ Harvard University, Cambridge, Massachusetts 02138
}

\begin{abstract}
In absolute identification of intensity, signals near the edges of the range being used are usually identified more accurately than those in the midrange. In one account, the extreme signals serve as anchors, and judgments are postulated to deteriorate as the distance from the signal to the nearest anchor increases. Our data suggest that, provided one corrects for the inherent asymmetry of errors for end and interior signals, the edge effect is rather smaller than it might first appear and is largely confined to the more intense edge. Moreover, anchors are not necessarily located at the edges of the range, but rather at the edges of the largest subset among which difficult discriminations are required. Further, this subset is not defined wholly by the signals used in a particular run, but by these together with those previously encountered in that day's session. Neither practice nor payoffs appear to influence the location of the anchor so long as the discrimination requirements are maintained. Finally, the role of anchors is interpreted in terms of the differential location of an attention band which controls the sample size upon which the representation of the signal is based.
\end{abstract}

In the absolute identification (AI) of intensity, a subject typically performs better on signals near the extremes of the intensity range than on interior signals. Durlach and Braida (1969) called this phenomenon a "resolution edge effect." One explanation of it is that subjects used the extreme signals as reference points or anchors and that judgments deteriorate as a function of intensity distance from the anchors. Our purpose here is, first, to examine empirically some of the factors that appear to control anchor locationit is not always as simple as the most extreme signaland, second, to propose that the explanation of the resolution edge effect in terms of distance from anchors follows naturally from the hypothesis that attention bands exist for intensity (Green \& Luce, 1974).

\section{GENERAL METHOD}

\section{Basic Al Procedure}

Before each block of 100 trials, the signals were presented twice in ascending order of intensity together with a visual display identifying their ranks in the signal set. Following this, each trial consisted of a warning interval, a 500-msec signal interval, and a response interval; all intervals were marked by unambiguous visual displays. Response buttons corresponded in a natural order to the signal set. Feedback, consisting of the actual rank of the signal, was provided after all (two or three) of the subjects had responded.

Each observer received a summary of his performance during the brief break following each block of 100 trials. Somewhat longer breaks occurred after every 300 trials. Typically, 1,200 trials were completed in a daily 2 -h session.

All experimental operations were controlled by a PDP-15 computer. The observers were seated in sound-attenuating chambers

This research was supported in part by National Science Foundation grants. and listened to binaural stimuli presented through TDH-39 earphones.

\section{Outline of Experiments}

Table 1 outlines the experimental procedures.

Column 1: Experiments.

Column 2: The Al procedure was described above; the RI and 4FSP paradigms will be described under Experiment 4.

Column 3: The pulse-train signals consisted of $10-\mu \mathrm{sec}$ pulses generated every $1 \mathrm{msec}$, so this signal had the same period as the $1,000-\mathrm{Hz}$ sinusoid one, which was designated "sinusoid" in the outline. Both the white noise and the pulse train were low-pass filtered at $8,000 \mathrm{~Hz}$, and the sinusoid signal was passed through a $100-\mathrm{Hz}$-wide band-pass filter centered at $1,000 \mathrm{~Hz}$.

Column 4: The number of different intensities used in the experiment is listed.

Column 5: For some ranges, more than one set of absolute intensities was tested. On each line in Row 4, the first number states a range in decibels and the numbers following the colon are each of the maximum intensities in decibels tested for that range. Each range appears on a separate line.

Column 6: Distribution A split the signals into two groups of equal size with successive signals in each group separated by $3 \mathrm{~dB}$. For example, in Experiment $5 \mathrm{~b}$ with a $54-\mathrm{dB}$ range and a maximum at $81 \mathrm{~dB}$, the 10 intensities were (in descending order) $81,78,75,72$, 69 and 39, 36, 33, 30, $27 \mathrm{~dB}$. Distribution B involved three pairs of signals, with the members of each pair separated by $3 \mathrm{~dB}$ and with the pairs located at the minimum, center, and maximum intensities of the range. Distribution $C$ was equal decibel spacing over the range.

\section{EXPERIMENT 1}

\section{Procedure}

The effect of range upon Al performance was investigated for three different types of signals: pulse train, white noise, and a $1,000-\mathrm{Hz}$ sinusoid. In each case, four values of intensity were to be absolutely identified. The intensities in the lower and upper pairs of signals were separated by $3 \mathrm{~dB}$ for all ranges; thus the separation between the middle two intensities is equal to the range minus $6 \mathrm{~dB}$. In order not to confound the effect of range with absolute intensity, the smaller ranges were run at several levels of intensity. 
Table 1

Outline of Experiments (see text)

\begin{tabular}{|c|c|c|c|c|c|c|c|}
\hline $\begin{array}{c}\text { Experi- } \\
\text { ment }\end{array}$ & Procedure & $\begin{array}{c}\text { Stimulus } \\
\text { Type }\end{array}$ & $\begin{array}{c}\text { Number of } \\
\text { Stimuli }\end{array}$ & $\begin{array}{l}\text { Range: Maximum } \\
\text { Intensity or } \\
\text { Intensities }\end{array}$ & $\begin{array}{c}\text { Intensity } \\
\text { Distribution }\end{array}$ & Observers & $\begin{array}{c}\text { Trials } \\
\text { per Observer } \\
\text { per Datum }\end{array}$ \\
\hline $1 \mathrm{a}$ & AI & Pulse Train & 4 & $\begin{array}{l}16: 63 \\
26: 48,68,88 \\
36: 73 \\
46: 68,78,88 \\
56: 83 \\
66: 88,88\end{array}$ & A & P.G., A.G., L.A. & 600 \\
\hline $1 \mathrm{~b}$ & $\mathrm{AI}$ & Noise & 4 & $\begin{array}{l}16: 43,83 \\
36: 63,83 \\
48: 83\end{array}$ & A & P.G., A.G., L.A. & 3000 \\
\hline $1 \mathrm{c}$ & Al & Sinusoid & 4 & $\begin{array}{l}18: 63,93 \\
33: 78,93 \\
48: 93\end{array}$ & A & P.G., A.G., L.A. & 300 \\
\hline 2 & Al & Noise & 6 & $63: 93$ & B & J.G., S.T., D.W. & 2400 \\
\hline 3 & AI & Sinusoid & 6 & $\begin{array}{l}33: 63 \\
63: 93\end{array}$ & B & D.M., J.C. & 1200 \\
\hline $4 a$ & RI & Sinusoid & 6 & $6: 63^{*}$ & $C^{*}$ & J.K., D.Y. & 600 \\
\hline $4 b$ & 4RSP & Sinusoid & 6 & $63: 93$ & B & J.K., D.Y. & 2400 \\
\hline $4 c$ & AI & Sinusoid & 6 & $63: 93$ & B & J.K., D.Y. & 2400 \\
\hline $5 a$ & AI & Sinusoid & 5 & $12: 39,54,69,84$ & $\mathrm{C}$ & P.T., D.R. & 1200 \\
\hline $5 \mathrm{~b}$ & AI & Sinusoid & 10 & $\begin{array}{l}27: 54,84 \\
39: 75 \\
42: 69 \\
54: 81\end{array}$ & A & P.T., D.R. & 1200 \\
\hline $5 \mathrm{c}$ & AI & Sinusoid & 10 & $54: 81$ & $\mathrm{C}$ & P.T., D.R. & 2100 \\
\hline
\end{tabular}

${ }^{*}$ Stimuli requiring identification were $57,58,59,61,62,63 \mathrm{~dB}$ SPL. An additional 12 stimuli, $30-36 \mathrm{~dB}$ and $82.93 \mathrm{~dB}$, were presented but did not require a response. See description of RI procedure in text.

The type of signal remained the same throughout a session, but the range and absolute intensities were varied from block to block according to a schedule which balanced their order within each session. All conditions for one type of signal were completed before the introduction of a new type; the order was pulse train, noise, and sinusoid.

\section{Results}

Figure la shows the percentage of correct identifications as a function of intensity for the pulse trains (Experiment 1a). Solid lines connect paired signals. All four data points from each condition have identical symbols, and those for conditions with the same range have symbols of similar shapes, e.g., those from a 66-dB range are all represented by downward-pointing triangles. Figures $1 \mathrm{~b}$ and $1 \mathrm{c}$ show similar representations of the results of Experiments $1 \mathrm{~b}$ and $1 \mathrm{c}$, respectively.

For these sets of four signals, there is no confusion between upper and lower pairs; all the confusions are within these pairs. For the 42 pairs shown in Figure 1, performance on the extreme member within each pair, either Signal 1 or Signal 4, was better in about half $(20 / 42)$ of the cases. Hence there is no evidence of a response bias to pick the extreme stimulus in this data.

The mean percentage of correct identifications per signal pair is shown (Figure 1d) as a function of the mean of the intensities in the pair for all three types of signals. The same pattern results for each range, and so simply the mean across range is shown. The three curves are similar and, taken together, exhibit an overall edge effect in the sense of better performance near 20 and $90 \mathrm{~dB}$ than in the midrange. ${ }^{1}$ The similarity in performance near $45 \mathrm{~dB}$ is remarkable, since this intensity occurred in the midrange for the pulse train and noise signals but as the minimum intensity for the sinusoid signal.

\section{EXPERIMENT 2}

\section{Procedure}

We employed both extensive practice and a payoff matrix in an attempt to induce equal performance throughout the intensity range. The signals were white noise at $30,33,60,63,90$, and $93 \mathrm{~dB}$ SPL. Over 12,000 trials were run as practice before the finalperformance data were collected. The payoff matrix offered a $\$ .50$ reward per block for each percentage point increase on center pair performance balanced by a $\$ .10$ penalty for each percentage point decrease in performance on the extreme pairs; no other aspect of performance was rewarded or penalized.

\section{Results}

The entires in Table 2 are the percentages of correct identifications for the three pairs of intensities on the initial and final 2,000 trials of practice and under the 


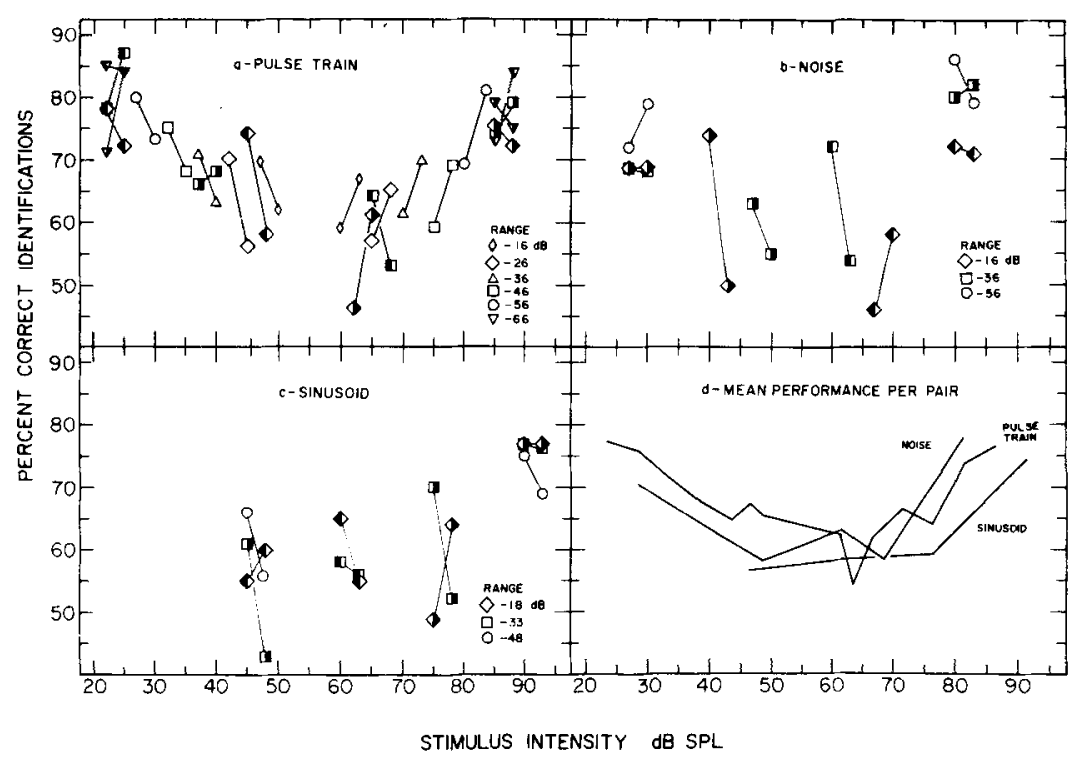

Figure 1. Percentages of correct identifications at each signal intensity in Experiment 1 are given for pulse-train (1a), noise (1b), and sinusoid (1c) signals. Solid lines connect intensities in a pair of signals. Identical symbols are used for all four signals in each condition. All conditions at a fixed range have similar symbols (in legend). For each type of signal, Figure 1d shows the mean percentages of correct identifications per intensity pair as a function of the mean of the intensities in the pair.

payoff matrix. Confusions between pairs did not occur after the first practice session. An edge effect is consistently apparent: performance is better on the extreme pairs than on the center pair.

The 12,000 trials of practice led to some improvement on the center pair, 7 percentage points on the average, but even greater improvement occurs on the low pair (10 percentage points) and the edge effect is not appreciably diminished. The further inducement of differential payoffs had no effect. Lippman, Braida, and Durlach (1976), in a more extensive investigation, also found payoffs to have no effect on sensitivity.

Table 2

Effect of Practice and Payoff Matrix

\begin{tabular}{llccc}
\hline & & \multicolumn{3}{c}{ Experiment 2 } \\
\cline { 3 - 5 } & & \multicolumn{3}{c}{ Pair Intensity } \\
& & 30,33 & 60,63 & 90,93 \\
\hline J.F. & I & 70 & 64 & 87 \\
& F & 80 & 60 & 89 \\
& P & 80 & 63 & 89 \\
S.T. & I & 76 & 55 & 90 \\
& F & 84 & 65 & 95 \\
& P & 84 & 68 & 94 \\
D.W. & I & 67 & 50 & 80 \\
& F & 78 & 64 & 80 \\
& P & 77 & 62 & 88 \\
Mean & I & 71 & 56 & 86 \\
& F & 81 & 63 & 88 \\
& P & 80 & 64 & 90 \\
\hline
\end{tabular}

Note-Percentage of correct identifications per stimulus pair are shown for the initial (I) and final $(F)$ 2,000 trials of practice. The payoff $(P)$ condition followed the practice trials.

\section{EXPERIMENT 3}

\section{Procedure}

Both Experiments 1 and 2 introduced observers to the maximum range at the beginning of the experiment. By contrast, in this experiment the observers received 7,000 trials of experience on the 6-signal AI using a 1,000- $\mathrm{Hz}$ sinusoid at $30,33,45,48,60$, and $63 \mathrm{~dB}$ SPL before having any experience with the larger (30-93 dB) range at the same intensities used in Experiment 2. We wished to see how prior experience in which the $60,63-\mathrm{dB}$ signals were an extreme pair would affect performance when they later appeared in the interior of a larger range.

\section{Results}

The percentage of correct identifications per intensity pair is our measure of performance in Table 3 , which shows both individual and mean performance for Experiment 3. For the initial phase of the experiment, where the $60,63-\mathrm{dB}$ pair is the maximum, the effect of practice is similar to that in Experiment 2: improvement occurs on the center pair, but the improvement on the low pair is greater and the edge effect is not significantly decreased. When the range was then increased and the $60,63-\mathrm{dB}$ pair became interior to the range, performance on this pair dropped by more than 10 percentage points.

\section{EXPERIMENT 4}

\section{Procedure}

To investigate how experimental procedure influences the anchor location, different response conditions were employed for $1,000-\mathrm{Hz}$ sinusoid signals with intensities near 30,60 , and $90 \mathrm{~dB}$ SPL.

Experiment $4 a^{2}$ At the beginning of each trial, three preliminary signals, with intensities randomly selected from a set of 12 (6 near $30 \mathrm{~dB}, 6$ near $90 \mathrm{~dB}$ ), were presented but required no response. 
Table 3

Effect of Practice

\begin{tabular}{llcccc}
\hline & & \multicolumn{4}{c}{ Experiment 3 } \\
\cline { 3 - 5 } & & \multicolumn{4}{c}{ Pair Intensity } \\
& & 30,33 & 45,48 & 60,63 & 90,93 \\
\hline J.C. & I & 55 & 59 & 72 & \\
& F & 70 & 59 & 79 & \\
& LR & 65 & & 60 & 86 \\
D.M. & I & 57 & 53 & 73 & \\
& F & 63 & 62 & 70 & \\
& LR & 62 & & 61 & 82 \\
Mean & I & 58 & 56 & 73 & \\
& F & 66 & 60 & 74 & \\
& LR & 64 & & 61 & 84 \\
\hline
\end{tabular}

Note-Percentage of correct identifications per stimulus pair are shown for the initial (I) and final (F) 2,000 trials of practice with the 60,63-dB maximum. The large range (LR) condition used this pair as interior stimuli.

The test signal was then presented, observers responded either "low" or "high," and feedback was given. "Low" test intensities were 57,58 , or $59 \mathrm{~dB}$, "high" test intensities were 61,62 , or $63 \mathrm{~dB}$. Thus observers made a relative identification (RI) of the test signal with respect to the median of $60 \mathrm{~dB}$ (which was never presented). Although the test intensities covered only a 6-dB range, the preliminary signals generated a $63-\mathrm{dB}$ range.

Experiment $4 b$ used three pairs of intensities: $30,33,60,63$, and $90,93 \mathrm{~dB}$. The observers were not required to discriminate between the intensities within the extreme pairs; they merely identified them as low or high extremes. The center pair of signals was to be absolutely identified. Thus, four responses (4RSP) were required: "low pair," "Signal 3," "Signal 4," and "high pair."

Experiment 4c was a conventional 6-signal AI at the intensity values of Experiment $4 \mathrm{~b}$. Unlike $4 \mathrm{~b}$, the observers were required to discriminate between the intensities in the extreme pairs as well as the middle pair.

\section{Results}

Performance is given in terms of percentage of correct identifications per intensity pair (Table 4). The values in the RI volumn are the PC values corresponding to the average $d^{\prime}$ values obtained for the two 3-dB separations bracketing the 60-dB standard ( 58 vs. 61 and 59 vs. $62 \mathrm{~dB}$ ). The 4 RSP column gives performance only for the $60,63-\mathrm{dB}$ pair; identification of low and high pair was $100 \%$. Average performance

Table 4

Effect of Experimental Procedure

\begin{tabular}{|c|c|c|c|c|c|}
\hline & \multicolumn{5}{|c|}{ Experiment (Procedure) } \\
\hline & \multirow{2}{*}{\multicolumn{2}{|c|}{$\frac{4 \mathrm{a}(\mathrm{RI})}{\text { Pair }} \frac{4 \mathrm{~b}(4 \mathrm{RSP})}{\text { Pair }}$}} & \multicolumn{3}{|c|}{$4 \mathrm{c}(\mathrm{AI})$} \\
\hline & & & \multicolumn{3}{|c|}{ Pair Intensity } \\
\hline & $60,63^{*}$ & 60,63 & 30,33 & 60,63 & 90,93 \\
\hline $\begin{array}{l}\text { D.Y. } \\
\text { D.K. }\end{array}$ & $\begin{array}{l}66 \\
72\end{array}$ & $\begin{array}{l}67 \\
78\end{array}$ & $\begin{array}{l}65 \\
73\end{array}$ & $\begin{array}{r}58 \\
66\end{array}$ & $\begin{array}{l}75 \\
83\end{array}$ \\
\hline Mean & 69 & 73 & 69 & 62 & 79 \\
\hline
\end{tabular}

Note-Entries are percentage of correct identifications per stimulus pair. 'Estimated from average d', see text. in the RI condition appears to be slightly poorer than for the 4RSP experiment; this difference is due to one of the two subjects.

Both subjects show a decrease in performance when increased discrimination is required at the extremes of the stimulus range. This decrease in performance is roughly the same magnitude as that seen in Experiment 3 when the $60,63-\mathrm{dB}$ pair is used at the interior rather than at the extreme of the range; moreover, the absolute levels for the corresponding conditions are approximately the same.

\section{EXPERIMENT 5}

\section{Procedure}

Experiment 5a was a 5 -signal AI using a $1,000-\mathrm{Hz}$ sinusoid with a 3-dB separation between intensity values. We examined performance in this task for four different levels of absolute iniensity chosen so that no intensity was used for more than one level. The intensities lay between 27 and $84 \mathrm{~dB}$ SPL.

Experiment 5b used combinations (see Figure 3) of the five intensities in Experiment 5a for a 10-signal AI task. The range varied between 27 and $54 \mathrm{~dB}$.

Experiment 5c examined performance in a 10-signal task with a 6- $\mathrm{dB}$ difference between successive signals (a 54-dB range). The maximum intensity, $81 \mathrm{~dB}$, was the same as that used for the 54-dB range in Experiment $5 \mathrm{~b}$.

\section{Results}

Interior signals may be confused with signals which are either more or less intense, whereas extreme signals can be confused with interior signals that lie on just one side of the extreme. This alone must account for some part of the edge effect. The following relatively atheoretical adjustment procedure corrects for this source of differences.

The only errors on the minimum intensity signals are confusions with more intense signals, and so the comparable measure for interior signals treats only confusions with more intense signals as errors; confusions with less intense signals are treated as correct identifications for this purpose. The corresponding adjustment for comparison with the maximum intensity signal only treats confusions with less intense signals as errors. Obviously, applying this correction to an extreme signal does not change the percent correct measure, whereas performance on the interior signals necessarily increases. ${ }^{3}$

The results for the two observers are shown in Figure 2 with the unadjusted and adjusted percentage of correct identifications represented by open and closed symbols, respectively. For each set of five intensities, adjustments are made with respect to the minimum for the three least intense signals and to the maximum for the three most intense signals; as mentioned above, this does not change the values at the two extremes. Note that the center signal appears twice, being adjusted in both directions. Good agreement between the two adjusted values suggests that there are no criteria or distribution irregularities 


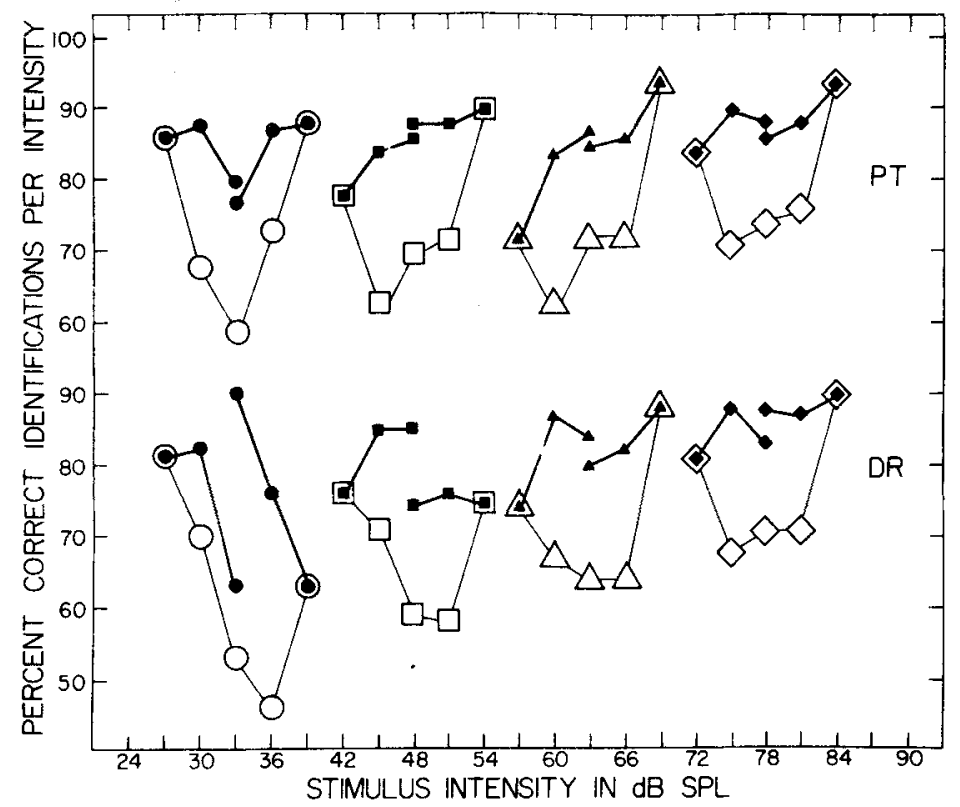

Figure 2. Percentages of correct identifications at each signal intensity for both subjects in Experiment 5a are shown as open symbols; closed symbols give the adjusted performance measure described in the text. Solid lines connect the five signals in each set of intensities.

invalidating the procedure. The large difference in D.R.'s adjusted score for the 33-dB signal indicates a strong bias to overestimate intensity.

The unadjusted scores show pronounced edge effects, although performance at the maximum intensity is generally greater than that at the minimum. Except for the absolute minimum intensity, $27 \mathrm{~dB}$, the adjusted scores show no edge effect whatsoever at the relative minimum intensity. Evidently, the edge at the minimum intensity seen in the unadjusted scores is attributable entirely to response bias. The edge effect at the maximum intensity survives this treatment, although it is much reduced. A reanalysis of the data reported in Luce, Green, and Weber (1976) reveals a similar edge effect for the maximum intensity for a range of $13.5 \mathrm{~dB}$. These results are not inconsistent with the reduction in the edge effect for decreasing range reported by Berliner, Braida, and Durlach (1977), although their data show no edge effects for any range less than $18 \mathrm{~dB}$.

A similar relation between adjusted and unadjusted scores is seen in Experiment $5 \mathrm{~b}$ (Figure 3). When the two groups of five signals are separated by more than $10 \mathrm{~dB}$, no confusions exist between groups, and so we treat each group separately and adjust as above. When the separation between groups is $3 \mathrm{~dB}$, the eight least intense signals are adjusted with respect to the minimum and the eight most intense signals with respect to the maximum, producing two estimates for the six central signals. In the unadjusted measure, edge effects appear for both the minimum and maxi- mum intensity in each 5-signal group (when the groups are separated by more than $15 \mathrm{~dB}$ ). Put another way, an edge effect is apparent for both extrema of the 10-signal set and for the interior boundaries of the two 5-signal groups. In the adjusted measure, the interior edge effects disappear, leaving only the edge effects at the two extrema.

The effect of stimulus distribution on performance is shown in Table 5. The overall performance measured in bits of information transmitted is compared for Experiment $5 \mathrm{c}$ and for that condition of Experiment $5 b$ having the same range and maximum. These two conditions were examined on the last 2 days of Experiment 5 . When the signals are spaced equally in decibels (Experiment $5 \mathrm{c}$ ), the overall performance is somewhat better $(.05<\mathrm{p}<.1)$ than the grouped distribution (Experiment $5 \mathrm{~b}$ ).

\section{DISCUSSION}

A simple Thurstonian model, in which each signal is represented as a random variable with a mean that depends upon the signal value but otherwise having the same distribution, is inadequate as a general predictor of AI performance. In such a model, as signal separation increases, the distance between signal representations increases and performance improves until it becomes perfect, i.e., until information transmitted equals input information. In his classic paper, Miller (1956) reviewed many experiments showing that perfect performance can only be obtained in one 


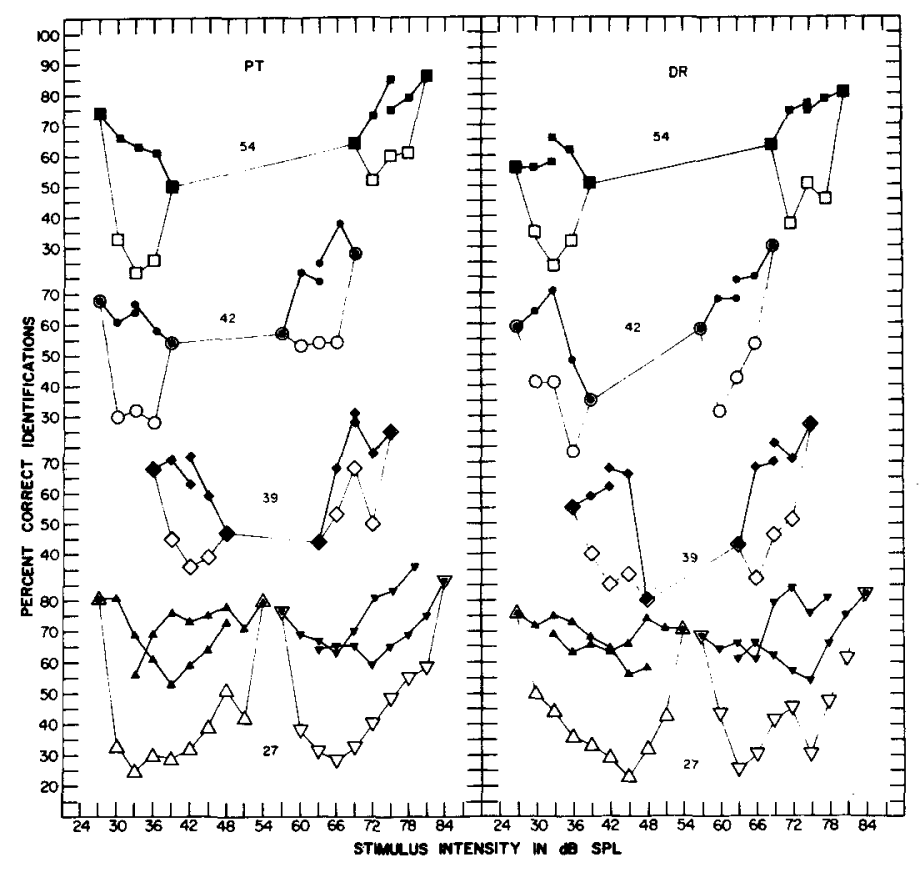

Figure 3. Percentages of correct identifications at each signal intensity in Experiment 5b are shown as open symbols; closed symbols give the adjusted performance measure described in the text. Solid lines connect the 10 signals in each set of intensities. The data from different ranges (54 to $27 \mathrm{~dB}$ ) are staggered for clarity. In conditions DR-42 and PT-54, the adjusted values for the middle signal are identical and so appear as one point.

dimension when the number of identified signals is small, about $7 \pm 2$. For more signals, performance asymptotes at from 2.3 to 3.2 bits of information transmitted, regardless of input information or signal separation.

Durlach and Braida (1969) and Gravetter and Lockhead (1973) have independently suggested that the variance of the assumed distribution is linearly related to the square of the signal range. Durlach and Braida define range to be the difference, in bels, between the smallest and the largest signals, whereas Gravetter and Lockhead define it to be the difference, in decibels, between the smallest and the largest criterion. Usually the smallest (largest) criterion lies between the two least (most) intense signals. This modified Thurstonian model-the range modelcorrectly predicts the observed asymptotic performance, but it does not predict certain common results, e.g., edge effects, and is contradicted by other results shown below.

Table 5

Effect of Distribution on Performance Measured in Bits of Information Transmitted

\begin{tabular}{ccc} 
& \multicolumn{2}{c}{ Experiment (Distribution) } \\
\cline { 2 - 3 } & $5 \mathrm{~b} \mathrm{(B)}$ & $5 \mathrm{c}(\mathrm{C})$ \\
\hline P.T. & 1.76 & 1.90 \\
D.R. & 1.51 & 1.73 \\
\hline
\end{tabular}

\section{Failures of the Range Modification}

For a fixed subset of signals, the range model predicts that they should be correctly identified less often as other signals are changed so as to increase the overall range. This follows because the means of the representations of signals in the subset maintain a fixed relationship, but their variances are increased by increasing the range. However, performance on the subsets of extreme signals (signals near 30 and $90 \mathrm{~dB}$ ) in Experiments 1 and 5 reveals no such decrease; in fact, subset performance and range are not ordered in any way.

Furthermore, given that the range determines the variance, the distribution of signals within that range should not affect performance (Braida \& Durlach, 1972). This prediction is contradicted by the results of Experiment 5, where better performance was obtained with equally spaced signals than when signals were grouped near the extremes. Both Braida and Durlach (1972, Experiment 2) and Gravetter and Lockhead (1973, Experiment 3) also found that differences in distribution result in a differences in performance.

Finally, when discrimination is not required within the pairs of signals at the extremes, performance on the center pair is better than when such discrimination is required (Experiment 4). For Braida and Durlach's (1972) model, however, the mean value of the signal representation depends only upon intensity 
and the variance depends only on range, implying that the identical sets of signals should have identical representations; hence performance on the center pair should be the same. The direction of the observed difference is predicted by the model of Gravetter and Lockhead, which assumes range is determined by the extreme criteria rather than the extreme signals.

\section{The Anchor Model}

Berliner and Durlach (1973) modified the original model to account for such data. They suggested that the representations of certain signals may be used as reference points in making judgments. It is the distance from these "anchors" that limits performance. With equal spacing of signals, this distance and range are confounded, permitting good predictions of overall performance from the preliminary model.

The anchor model provides a more satisfactory account of the data. Edge effects result from using the extreme signals as anchors, since performance for interior signals is reduced by the larger variance of their representations. The distribution of signals is important because it is the distance from an anchor, not the range, which limits performance. And given that the extremes are anchors, performance on a set of signals with a fixed intensity relation to that extreme should not vary with range, since the distance to the nearest anchor remains constant.

\section{Factors in Anchor Location}

The above discussion, then, suggests the general rule that anchors are located at extreme signals. Other of our data suggest that a slight revision in the rule is needed.

In the initial part of Experiment 3, the $60,63-\mathrm{dB}$ pair is the maximum of the range, and, consistent with the above rule, an anchor should be located there. When the $90,93-\mathrm{dB}$ pair is introduced as the maximum, performance on the $60,63-\mathrm{dB}$ pair deteriorates; presumably the anchor has been relocated at the new maximum. Performance for the $60,63-\mathrm{dB}$ pair is approximately the same in the initial phase of Experiment 3 and in Experiments $4 a$ and $4 b$. It is reduced by approximately the same amount, 10 percentage points, in the second phase of Experiment 3 and in Experiment $4 c$, which use the same intensities. Since the same performance arises in the corresponding conditions in the two experiments, it seems reasonable to try to attribute the decreases in performance to the same cause, namely a change in the location of the anchor. But there is a problem, since throughout Experiment 4 the range was from 30 to $90 \mathrm{~dB}$.

A possible resolution is to suppose that the anchors are not necessarily located at the extreme signals presented, but rather at the most extreme signals of the set for which fine discrimination is required. Given that the extreme signals require no response or need only be identified as extreme, it is inefficient to use these extremes as anchors; when all judgments can be made relative to the center of the range, the anchor appears to be located there and not at the extremes.

The overall edge effect (better performance near 30 and $90 \mathrm{~dB}$ seen in Figure 1) in Experiment 1 suggests that the anchors are actually located at the most extreme intensities encountered during the day, and they are used even when these intensities are not present in a particular block of trials. Such an effect would be greatest in Experiment 1 because the absolute maxima occurred during each session. When only one range of intensities is tested during a session, as in Experiment 5, this effect is reduced (Figure 3), but may be responsible for the absence of an edge effect for local minimum intensities whose absolute value is greater than the absolute minimum (Figure 2). In this experiment, the absolute minimum was present in the initial session, whereas the local maximum was successively increased until the absolute maximum was achieved in the final sessions. Thus each local maximum was at least as intense as all previously presented maxima (and edge effects are seen for all), whereas no minimum was ever less than the initial one, and no edge effects appear for minima greater than the absolute minimum. The maintenance of anchors across sessions could account for the absence of a minimum edge effect for the sinusoid signal in Experiment 1 . The minimum intensity for this signal was an interior intensity for the signal types preceding it.

The absence of a local edge effect (better performance on the interior member of the high or low pair seen for about half the pairs in Figure 1) in Experiment 1 might suggest that the interior signals were being used as anchors. However, when discrimination is required at the extremes of the stimulus range, it appears that interior signals cannot be used as anchors despite practice (Experiments 2 and 3), payoffs (Experiment 2), or previous use of the signals as anchors (Experiments 3 and 4). Experiment 5 b shows no such anchoring (no interior edge effect in Figure 3), even though the groups are widely separated and the interior signals are used as anchors when the group is presented alone. Together, these results suggest that interior signals cannot be used as anchors, and so some other interpretation of Experiment 1 is needed.

If the anchor is located between the two signals at each extreme, rather than at either signal representation, then it is a matter of chance for which of the two signals performance will be better. In Experiment 1 , the probability of performing better on the extremes than on the interior signals is at this chance level $(48 \%)$. This anchor location corresponds to the criteria location suggested by Gravetter and Lockhead (1973). Their model does not, however, employ the 
concept of anchors as described here; it is essentially a range model.

\section{Attention Band Hypotheses}

An alternative approach to increasing variance as a function of distance from an anchor was suggested by Green and Luce (1974). If we assume that the variability of the signal representation arises from some sort of sampling process of neural information-for present purposes, it doesn't matter what the details are-then the variability will depend, in part, on the size of the sample use, i.e., the variance of the representation will change in proportion to the sample size, other factors being equal. They suggested that attending to a sensory event amounts to locating one's neural sampling resources primarily at one region (band in one dimension) of the sensory space, so that signals occurring in that region are represented by a large sample size whereas those falling outside the region are represented by a much smaller one. Thus, in the Thurstonian model, the former have a representation with a much smaller variance than the latter. The term "attention band" need not imply conscious operation, and the failure of payoffs to affect anchor location suggests that the attention band location is not under the observer's control. Attention refers, instead, to the general notion of a process which selects some portion of the input for more intensive analysis; one example of this is the critical band concept (Fletcher, 1940) in frequency analysis.

The process of anchoring is easily interpreted in terms of this model; anchors are intensities at which the attention band tends to be located. The above discussion of anchor location can be interpreted as defining properties of attention band location. For example, edge effects indicate that the attention band tends to be located near the extreme intensities. That the edge effect appears as a continuous increase in performance as the distance to an edge decreases is not incompatible with this model, even though the attention band predicts only two discrete values for the variance. Given only two discrete variance values at one instant, if the attention band fluctuates some in location over time, as is suggested by the results of magnitude estimation (Luce \& Green, 1974), then the change in variance, viewed over time, would appear continuous.

While the attention band provides a plausible mechanism for the anchor operation, it should not be thought to be an extension of the modified range model. That range, or distance to an anchor, should directly affect intensity coding seems intuitively unsatisfactory and artificial. It seems to us more straightforward to treat range and anchor effects as arising from processes governing the location of the attention band. But these are a priori considerations, not subject to empirical verification by data such as these. Both models appear adequate to account for these data. Differences may, however, be found in the trial-to-trial predictions of the models, a topic we are currently investigating.

\section{CONCLUSION}

Regardless of the theoretical interpretation given to anchors, the following observations are supported by these data:

(1) The apparent edge effect is markedly reduced - eliminated almost entirely at low intensities and small ranges-by correcting for the asymmetries of errors on extreme vs. interior signals.

(2) Range alone is not the critical variable in $\mathrm{AI}$ performance; distance from an extreme signal (anchor) is usually a better predictor.

(3) Anchors tend to be located not at the most extreme signals, but somewhere within subgroups of the most extreme signals among which discrimination is required.

(4) The anchors near the absolute extremes encountered by the subject during a day tend to be used, even when these intensities are not present during a particular run.

(5) Neither practice nor bribes shift anchor location so long as discrimination requirements are maintained at that location.

\section{REFERENCES}

Berliner. J. E., \& Durlach, N. I. A perceptual-anchor model for context coding in intensity perception. Journal of the Acoustical Society of America, 1973, 54, 336(A).

Berliner, J. E., Durlach, N. I., \& Braida, L. D. Intensity perception. VII. Further data on roving-level discrimination and the resolution and bias edge effects. Journal of the A coustical Society of America, 1977, 61, 1577-1585.

BraIdA, L. D., \& DURLACH, N. I. Intensity perception. II. Resolu tion in one-interval paradigms. Journal of the Acoustical Society of America, $1972,51,483-502$.

Durlach, N. I., \& Braida, L. D. Intensity perception I. Preliminary theory of intensity resolution. Journal of the Acoustical Society of America, 1969, 46, 372-383.

Fletcher, H. Auditory patterns. Review of Modern Physics, 1940, 12, 47.65.

Gravetter, F., \& Lockhe ad, G. R. Criterial range as a frame of reference for stimulus judgment. Psychological Review, 1973, 80, 203-216.

GreEN, D. M., \& LUCE, R. D. Variability of magnitude estimates: A timing theory analys is. Perception \& Psychophysics, 1974, 15, 291-300.

Lippman, R. P., Braida, L. D., \& Durlach, N. I. Intensity perception $V$. Effect of payoff matrix on absolute identification. Journal of the Acoustical Society of A merica, 1976, 59, 129-134.

LuCE, R. D., \& GREEN, D. M. The response ratio hypothesis for magnitude estimation. Joumal of Mathematical Psychology, $1974,11,1-14$. 
LUCE, R. D., GreEN, D. M., \& Weber, D. L. Attention bands in absolute identitication. Perception \& Psychophysics, 1976, 20. 49-54.

Miller. G. A. The magical number seven, plus or minus two: Some limits on our capacity for processing information. Psychological Review, 1956. 63, 81-97.

\section{NOTES}

1. Note that percentage of correct responses computed for the extreme pairs is a nonparametric result. One could, of course, perform a Thurstonian analysis and compute $\mathrm{d}^{\prime}$ for each pair. Since no consistent response bias was apparent for these pairs, we expect $d^{\prime}$ and the percentage of correct responses to be mono- tonically related. Computations of $d^{\prime}$ do, in fact, show the same pattern as the data in Figure 1d.

2. We are indebted to Dr. Walt Jesteadt, who collected the data reported in Experiment $4 a$.

3. We prefer this nonparametric approach because it makes no assumption about the forms of the underlying densities. Performance measures based on a Thurstonian analysis are suspect when adjacent cells in the stimulus-response matrix are zero, which occurred in many of our conditions. When a Thurstonian analysis was possible, an examination of the sensitivity index, $d^{\prime}$, showed the same trends as those stated.

(Received for publication December 6, 1976; revision accepted June 2, 1977.) 